\title{
COADSORPTION OF BERYLLIUM AND POTASSIUM ON A (001) TUNGSTEN PLANE
}

\author{
Z. DWORECKI \\ Institute of Pliysics, Pedagogical University \\ Oleska 48, 45-052 Opole, Poland \\ (Received May 25, 1991)
}

\begin{abstract}
The adsorption and coadsorption of beryllium and potassium on the tungsten (001) plane was studied using a probe-hole field electron microscope (FEM). Measurements were made at $78 \mathrm{~K}$ for potassium and at $300 \mathrm{~K}$ for beryllium. It has been found that the adsorption of potassium decreases and that of beryllium increases the work function of the W(001) plane. At small coverages with potassium atoms $\left(\theta_{K}<0.2\right)$ on the $W(001)$ plane successive evaporation of beryllium atoms causes a decrease of the work function. At larger coverages with potassium atoms an opposite effect is observed. An attempt has been made to compare the experimental results with the theoretical models suggested recently.
\end{abstract}

PACS numbers: $61.55 . \mathrm{Hg}, 73.30 .+\mathrm{y}, 79.70 .+\mathrm{q}$

\section{Introduction}

Adsorptiom of beryllium and potassium on tungsten has been studied for many years by means of a variety of experimental techniques. A comprehensive list of the papers dealing with this problem can be found in Refs. [1-5].

In the present work the adsorption of beryllium and potassium on tungsten was investigated using the field electron emission technique. The purpose of this work was to investigate the changes in the work function caused by the evaporation of beryllium atoms onto the surface of tungsten precovered with potassium. The behaviour of beryllium deposited on the tungsten surface, clean or precovered with potassium, is interesting in view of its small atomic radius $(1.13 \AA)$ as compared with the atomic radii of potassium $(2.31 \AA)$ and tungsten $(1.37 \AA)$.

\section{Experimental}

The measuring apparatus and the experimental procedure have been described in [5]. The value of the work function for the clean (001) plane of tungsten is assumed to be $\varphi_{001}=4: 63 \mathrm{eV}[6]$. 


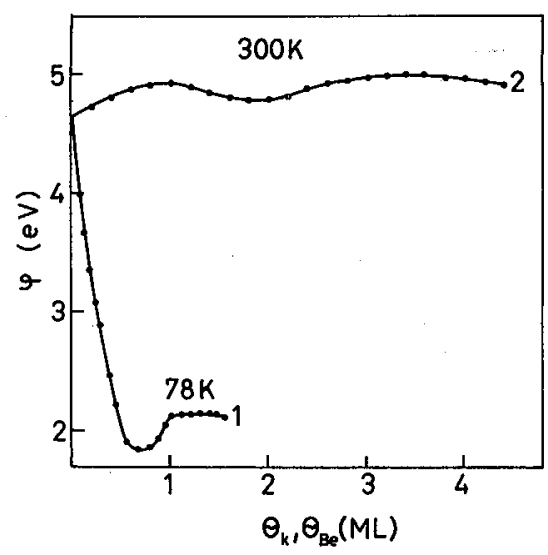

Fig. 1. Dependence of the work function $\varphi$ on the coverage of potassium $\theta_{\mathrm{K}}$, and beryllium $\theta_{\mathrm{Be}}$ deposited onto the (001) plane of tungsten. Curve 1 and 2 are concerned with the adsorption of potassium and beryllium, respectively.

\section{Results and discussion}

Figure 1 shows the dependencies of the work function $\varphi$ on the coverage $\theta$ for the adsorption of potassium and beryllium onto the (001) plane of tungsten. Curve 1 represents $\varphi$ versus $\theta_{\mathrm{K}}$ on the clean $\mathrm{W}(001)$ plane at $78 \mathrm{~K}$. Curve 2 is $\varphi$ versus $\theta_{\mathrm{Be}}$ on the clean $\mathrm{W}(001)$ at $300 \mathrm{~K}$. The coverage $\theta_{\mathrm{Be}}$ or $\theta_{\mathrm{K}}$ on the (001) plane of tungsten has been determined on the basis of the dependence of the work function on the evaporation time of beryllium or potassium atoms. It has been assumed in the case of beryllium that a monolayer is formed after a time of evaporation when the first maximum of the $\varphi=f(t)$ curve is achieved. In the case of potassium a monolayer is formed after the evaporation time needed to obtain the saturation of the $\varphi=f(t)$ curve. As it can be seen in curve $1, \varphi$ decreases initially with increasing $\theta_{\mathrm{K}}$, reaches its minimum value $\varphi_{\min }=1.79 \mathrm{eV}$ and then increases monotonically up to the saturation value $\varphi_{\text {sat }}=2.21 \mathrm{eV}$. The shape of the curve 1 is typical for the adsorption of potassium on tungsten and similar to the curves reported in $[2,3,5,7,8,9]$.

Theoretical models for the adsorption of alkali metals on the metallic surfaces are described in [10]. The models proposed by Lang [11] and by Muscat and Newns [12] predict the shape of the $\varphi(\theta)$ curve for coverages $0 \leq \theta \leq 1$ as well as the position and value of $\varphi_{\min }$ in good accordance with experimental data.

Curve 2 shows the plot of the work function $\varphi$ versus $\theta_{\mathrm{Be}}$. It can be seen that $\varphi$ increases to a first maximum $\varphi_{\max 1}=4.93 \mathrm{eV}$, then decreases and passes through a minimum at $\varphi_{\min }=4.80 \mathrm{eV}$. Thereafter, $\varphi$ increases again and reaches the second maximum $\varphi_{\max 2}=5.02 \mathrm{eV}$ after which it slowly approaches a final value $\varphi_{\text {sat }}=4.90 \mathrm{eV}$. The first maximum of $\varphi$ corresponds to the monoatomic coverage, at which the surface concentration of beryllium atoms becomes equal to the surface concentration of tungsten atoms on the $\mathrm{W}(001)$ plane $\left(10 \times 10^{14}\right.$ atoms $\left./ \mathrm{cm}^{2}\right)$. Beryllium atoms occupy positions in the centre of the square formed by tungsten 
atoms on this plane, which results in the smoothing of the face [13] and therefore the work function increases due to the Smoluchowski effect [14]. Further deposition of beryllium leads to the accupation of sites between the beryllium adatoms; the surface becomes rougher and the work function decreases to a minimum. Further deposition causes the formation of beryllium layers with an increasing density which leads to an increase of the work function and a second maximum. Subsequent depositions of beryllium on the tungsten tip lead eventually to the formation of beryllium crystals changing the geometry of the tip [15]. As it was expected, the shape of the $\varphi=f\left(\theta_{\mathrm{Be}}\right)$ curve for beryllium deposited on the W(001) plane is similar to the $\Delta \varphi=f(t)$ obtained in study of the adsorption of beryllium on the same tungsten plane at room temperature [16], although some minor quantitative differences are observed.

Figure 2 shows the dependence of the work function $\varphi$ on the coverage $\theta_{\mathrm{Be}}$, for the coadsorption of potassium and beryllium onto the (001) plane of tungsten. At small potassium coverages (curve 1 and 2) the work function decreases with

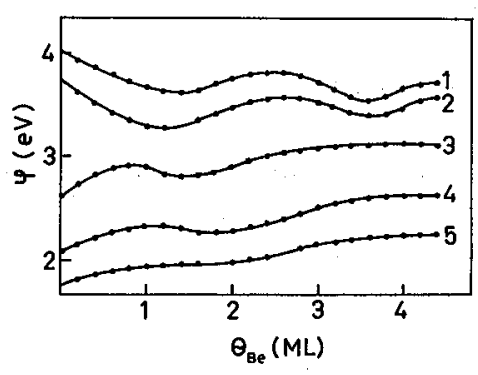

Fig. 2. Dependence of the work function $\varphi$ on the coverage of beryllium $\theta_{\mathrm{Be}}$ deposited on the potassium precovered (001) plane of tungsten. Curve 1 is at $\theta_{\mathrm{K}}=0.12 ; 2-$ $\theta_{\mathrm{K}}=0.16 ; 3-\theta_{\mathrm{K}}=0.36 ; 4-\theta_{\mathrm{K}}=1.00 ; 5-\theta_{\mathrm{K}}=0.66 \mathrm{C}$.

increasing $\theta_{\mathrm{Be}}$, passes through a first minimum, thereafter increases and reaches a flat maximum, next decreases to a second minimum and then increases to a saturation value. At larger potassium coverages (curves 3,4 and 5 ), the work function initially increases with increasing $O_{\mathrm{Be}}$ and, after reaching a maximum, decreases to a minimum value and then increases to a saturation value. The observed course of the $\varphi\left(\theta_{\mathrm{Be}}\right)$ curves can be explained by a mechanism of smoothing of the W(001) plane covered with potassium atoms, controlled by the mobility of the potassium on the surface of the tungsten tip kept at a temperature of $240 \mathrm{~K}$ during the evaporation of beryllium and next tempered at $400 \mathrm{~K}$. Since the value of the activation energy for migration of potassium atoms on tungsten reveals its maximum at the coverage $\theta_{\mathrm{K}} \approx 0.66$ [17], the corresponding change in the $\varphi\left(\theta_{\mathrm{Be}}\right)$ is minimal (curve $5)$. At this coverage the potassium layer atoms protrude over beryllium adatoms, i.e. surface segregation of potassium takes place. The second factor which can affect the observed work function changes may be the charge transfer from $\mathrm{K}$ atoms, but the experimental results obtained in the present work seem to indicate that the smoothing effect is prevailing. 
In recent years theoretical works were reported [18-21] about alkali metals alloyed on tungsten. It has been found that surface segregation of solute species occurs in such alloys. The theory predicted that the solute segregated layer in solid solution might be contracted when the Wigner-Seitz radius ratio is larger than $\sim 1.1$. In our case the ratio of the Wigner-Seitz radii of the two kinds of atoms deposited on the tungsten surface is much larger than $1.1\left(r_{s \mathrm{~K}} / r_{s \mathrm{Be}}=4.86\right.$ a.u./1.87 a.u.= 2.6). Therefore one should expect a large driving force for the segregation of the greatest species (K atoms) on the surface of the system studied. We will treat the adsorption of beryllium on potassium-precovered tungsten as the case of the adsorption of two solute species leading to a surface alloy of potassium and beryllium on tungsten. The form of the $\varphi\left(\theta_{\mathrm{Be}}\right)$ dependence as shown by curves 1-5 in Fig. 2 indicates the validity of the assumption of potassium protrusions over beryllium adatoms on the $\mathrm{W}(001)$ plane at small potassium coverages $\left(\theta_{\mathrm{K}}<0.2\right.$, curves 1 and 2). Potassium atoms starting from their positions in the centre of four tungsten atoms are displaced into saddle sites between tungsten atoms or over beryllium atoms. The displacement results in the roughness of the $W(001)$ plane which is revealed by decreasing work function. Also an opposite agent which causes an increase of the work function should be taken into account. Beryllium atoms can occupy the sites left by potassium atoms or other sites in the W(001) holes which leads to a smoothing effect of the crystal plane. At high potassium coverages (curves 3-5) the smoothing effect of beryllium atoms prevails over the influence of the surface segregation of potassium atoms with a slightly higher activation energy for surface diffusion on the $W(001)$ plane.

\section{Summary and conclusion}

From the data obtained using FEM some differences between adsorption of potassium and beryllium on the (001) plane of tungsten can be indicated. The steep decrease of the work function induced by potassium adsorption is associated with the transfer of negative charge from the adlayer into the substrate which results in the formation of a dipole layer. The behaviour observed for beryllium adsorption can be understood as a smoothing effect caused by placing beryllium atoms into holes existing on this crystal plane, which leads to an increase of the work function.

In the case of beryllium adsorption on the W(001) plane precovered with potassium the shape of curves $\varphi\left(0_{\mathrm{Be}}\right)$ results from two processes: displacement of potassium atoms over the deposited beryllium atoms and the smoothing of the $\mathrm{W}(001)$ plane by beryllium atoms. The process of surface segregation is assumed to accompany the adsorption of the two metals on the metal substrate. Potassium atoms may be displaced towards the surface, over beryllium atoms, because the Wigner-Seitz radius of potassium is much greater than that of beryllium atoms (is equal to 4.86 and 1.87 for potassium and beryllium, respectively. [22]). 


\section{Acknowledgements}

The author would like to thank Professor K.F. Wojciechowski and Dr. A. Kiejna for their helpful indication of the literature on the theory of the adsorption of metals and alloys.

The work was supported by the Research Project CPBP 01.08.A.

\section{References}

[1] E. Chrzanowski, E. Bauer, Surf. Sci. 173, 106 (1986).

[2] V.K. Medvedev, A.I. Yakivchuk, Fiz. Tverd. Tela 16, 981 (1974).

[3] R. Blaszczyszyn, M. Blaszczyszyn, R. Męclewski, Surf. Sci. 51, 396 (1975).

[4] Z. Sidorski, Appl. Phys. A 33, 213 (1984).

[5] Z. Dworecki, to be published in Surf. Sci.

[6] H.B. Michaelson, J. Appl. Plyys. 48, 4729 (1977).

[7] D.L. Felırs, R.E. Stickney, Surf. Sci. 24, 309 (1971).

[8] L.D. Schmidt, R. Gomer, J. Chem. Phys. 46, 1605 (1966).

[9] A.P. Ovtchinnikov, Fiz. Tverd. Tela 9, 628 (1967).

[10] A. Kiejna, K.F. Wojciechowski, Prog. Surf. Sci. 11, 203 (1981).

[11] N.D. Lang, Phys Rev. B 4, 4234 (1971); Solid State Commun. 9, 1015 (1971).

[12] J.P. Muscat, D.M. Newns, Solid State Commun. 11, 737 (1972); J. Phys. C 7, 2630 (1974).

[13] J. Polański, Z. Sidorski, Surf. Sci. 40, 282 (1973).

[14] R. Smoluchowski, Phys. Rev. 60, 661 (1941).

[15] Z. Dworecki, Acta Univ. Wratislav. Mat. Fiz. Astron. 439, 85 (1978).

[16] S. Zuber, Z. Sidorski, J. Polański, Surf. Sci. 87, 375 (1979).

[17] L.D. Schmidt, R. Gomer, J. Chem. Phys. 42, 3573 (1965).

[18] A. Kiejna, K.F. Wojciechowski, J. Phys. C, Solid State Phys. 16, 6883 (1983).

[19] A. Kiejna, J. Phys., Condens. Malter 2, 6331 (1990).

[20] H. Yamauchi, Phys. Rev. B 31, 7688 (1985).

[21] R.M. Digilov, W.A. Sozaev, Poverkhn., Fiz. Khim. Mekh. 7, 42 (1988).

[22] N.W. Ashcroft, N.D. Mermin, Solid State Physics, Holt, Rinehart and Winston, New York 1976, p. 62. 